\title{
PS-005 POLICY-DRIVEN INTERVENTIONS: TUBERCULOSIS
}

Gerhard Walzl, ${ }^{1}$ Novel Chegou, ${ }_{1}^{1}$ Stephanus Malherbe, ${ }^{1}$ Mark Hatherill, ${ }^{2}$ Thomas J. Scriba, ${ }^{3}$ Daniel E. Zak, ${ }^{4}$ Clifton E. Barry III, ${ }^{5}$ Stefan H.E. Kaufmann ${ }^{6}$.

${ }^{1}$ DSTINRF Centre of Excellence for Biomedical TB Research and MRC Centre for TB Research, Division of Molecular Biology and Human Genetics, Stellenbosch University, Tygerberg, South Africa; ${ }^{2}$ South African Tuberculosis Vaccine Initiative, Institute of Infectious Disease and Molecular Medicine \& Department of Paediatrics and Child Health, University of Cape Town, Cape Town, South Africa; ${ }^{3}$ South African Tuberculosis Vaccine Initiative, Institute of Infectious Disease and Molecular Medicine \& Department of Paediatrics and Child Health, University of Cape Town, Cape Town, South Africa; ${ }^{4}$ The Center for Infectious Disease Research, Seattle (WA) USA;

${ }^{5}$ Tuberculosis Research Section, Laboratory of Clinical Infectious Diseases, Division of Intramural Research, National Institute of Allergy and Infectious Diseases, National Institutes of Health, Bethesda (MD) USA; ${ }^{6}$ Department of Immunology, Max Planck Institute for Infection Biology, Berlin, Germany.

\subsection{6/bmjgh-2016-000260.5}

Annual tuberculosis $(\mathrm{Tb})$ rates decline by $1.7 \%$, which is inadequate to reach WHO targets. We describe three host-biomarker developments that are entering clinical testing and that could accelerate progress against TB.

Blood mRNA signatures with promising predictive ability for incident $\mathrm{TB}$ were recently described in African cohorts. Correlate of risk (COR) positive participants have 7 to 18 times increased risk for progression. A clinical trial is underway in South Africa under leadership of the South African Tuberculosis Vaccine Initiative to evaluate targeted chemoprophylaxis in COR positive people in an area with a very high prevalence of latent TB infection, where untargeted preventative treatment is not practical.

Historical data suggests that $85 \%$ of patients are cured after 4 months of $\mathrm{TB}$ treatment but attempts to shorten treatment without an unacceptable relapse rate have failed. Treatment shortening criteria based on PET/CT imaging and microbiological criteria were developed. An EDCTP/Bill \& Melinda Gates Foundation/NIH co-funded study, led by the Tuberculosis Research Section of the NIH, will start in South Africa and in China in 2017 to evaluate biomarker-driven treatment shortening to 4 months.

To address diagnostic bottlenecks, a blood-based 7-host marker diagnostic signature was recently found with promising screening potential for active TB. An EDCTP-funded project with Africa and EU partners aims to develop a point-of-care, finger stick blood test that can simultaneously measure all 7 markers and rule out $75 \%$ of people with symptoms compatible of active TB in whom the diagnosis is subsequently ruled out. Such screening tests could speed up diagnostic work-up and save significant costs. Taken together, biomarker-driven interventions are now being actively tested and hold promise to provide important tools towards the eventual eradication of the TB scourge. 